\title{
Cross-section analysis of coal workers' pneumoconiosis and higher brachial-ankle pulse wave velocity within Kailuan study
}

Yao Zheng ${ }^{3+}$, Lirong Liang ${ }^{2 \dagger}$, Tianbang Qin ${ }^{3}$, Guang Yang ${ }^{1}$, Shasha An ${ }^{1}$, Yang Wang ${ }^{1}$, Zhifang Li' ${ }^{1}$ Zhongda Shao ${ }^{3}$, Xiuping Zhu ${ }^{3}$, Taicheng $\mathrm{Yao}^{3}$, Shouling $\mathrm{Wu}^{1^{* \dagger}}$ and Jun Cai ${ }^{4^{*+}}$

\begin{abstract}
Background: Brachial-ankle pulse wave velocity (baPWV) is an independent predictor of cardiovascular events and mortality. However, there is no related data on the association of baPWWwith coal workers' pneumoconiosis (CWP). We explored the baPWV in subjects withCWP and the associated risk factors.

Methods: Thiscase-control study included 1,007 male CWP cases without a history of stroke and coronary heart disease and 1,007 matched controls from the Kailuan cohort study. All of the participants underwent assessment for baPWV and traditional cardiovascular risk factors. The cumulative silica dust exposure (work history linked to a jobexposure matrix) was estimated for the CWP cases.

Results: Compared with the controls, the CWP cases had higher baPWV $(1762.0 \pm 355 \mathrm{~cm} / \mathrm{s}$ vs. $1718.6 \pm 354 \mathrm{~cm} / \mathrm{s}, P=0$. 006) and a higher risk of increased baPWW (defined as more than the median baPWW of the population distribution; odds ratio $1.43,95 \%$ confidence interval 1.11-1.83) after adjusting for traditional cardiovascular risk factors. Age $\geq 60$ years, body mass index, heart rate, and hypertension were all significantly associated with increased baPW in the CWP cases. Compared to non-CWP subjects without hypertension, the odds ratios for increased baPW gradually increased (P for trend, 0.001) across the CWP subjects without hypertension (odds ratio 1.20,95\%confidence interval 0.90-1.61), subjects with hypertension alone (odds ratio 2.54, 95\% confidence interval 1.95-3.30), and CWP subjects with hypertension (odds ratio 3.34, 95\% confidence interval 2.56-4.37). We detected a significant positive exposure-response relationship between silica dust-exposure quartiles and increased baPWV in CWP cases ( $P$ for trend $<0.001$ ).
\end{abstract}

Conclusions: For patients with CWP, increased baPWW was associated with traditional cardiovascular risk factors and long-term silica dust exposure.

Keywords: Silica dust, Coal workers' pneumoconiosis (CWP), Arterial stiffness, Brachial-ankle pulse wave velocity

\section{Background}

Coal workers' pneumoconiosis (CWP) is a chronic occupational lung disease caused by long-term inhalation of coal dust. Given that CWP is the most common occupational disease in coal workers [1-3], the prevalence of CWP is usually higher in developing countries than in developed countries. In China, the prevalence of CWP has been previously reported to be $6.02 \%$ (95\% confidence interval (CI)

\footnotetext{
*Correspondence: drwusl@163.com; caijun7879@126.com

${ }^{\dagger}$ Equal contributors

${ }^{1}$ Kailuan Hospital, Tangshan, Hebei, China

${ }^{4}$ Department of Cardiology, Chaoyang Hospital, Capital Medical University, Beijing, China

Full list of author information is available at the end of the article
}

3.43-9.26\%), which is a rate that is higher than those reported for the United Kingdom (0.8\% for 1998-2000) and the United States (3.2\% for the 2000s) [4-6].

Most cases of CWP are caused by silica exposure. Adverse health effects from long-term, cumulative silica dust exposure (CDE) are an increasing public health concern worldwide. Recent studies have reported that long-term $\mathrm{CDE}$ increased the risk of death not only due to respiratory diseases but also due to cardiovascular diseases (CVDs), revealing the exposure-response relationships between cumulative silica dust exposure and mortality from CVDs $[7,8]$. These findings have increased the need to reduce the risk of cardiovascular 
mortality among patients with CWP. Since many CVDs can be prevented, early detection and prevention are key, particularly at the earlier stages of atherosclerosis. However, to our knowledge, no related research has been conducted among patients with CWP.

Arterial stiffness plays a critical role in the pathogenesis of atherosclerosis and cardiovascular events, is an independent predictor of cardiovascular mortality, and is a useful index in the prevention and early detection of CVD $[9,10]$. Among representative measures of arterial stiffness, brachial-ankle pulse wave velocity (baPWV), which reflects the stiffness of both the central and peripheral muscular arteries, has been frequently used as a simple and non-invasive measure of systemic arterial stiffness [11-13]. Moreover, many studies have demonstrated that baPWV is an independent predictor of cardiovascular events [14] and mortality in the general population and in patients with an increased risk of CVDs [15-17]. Although baPWV is a useful tool for identifying subpopulations at increased risk for CVD, no observational data have been reported that indicate whether increased baPWV is associated with CWP and whether potential risk factors for increased baPWV occur among patients with CWP. Therefore, the present study sought to explore these two issues in Chinese patients with CWP.

\section{Methods}

\section{Study population}

The present case-control study included participants with CWP from the Kailuan cohort study, which recruited 101,510 employees and retirees of the Kailuan (Group) Co. Ltd., a large coal company located in Tangshan City, Hebei province, China, from June 2006 to October 2007. Details of this prospective cohort study were described previously [18-20]. The study followed the guidelines of the Helsinki Declaration and was approved by the Ethics Committees of Kailuan General Hospital and Beijing Chaoyang Hospital, China. Written informed consent was obtained from all the participants.

A total of 16,185 coal workers were included at baseline and followed up with an examination for pneumoconiosis every 2-3 years. As of December 31, 2010, 1,806 cases of CWP were diagnosed, which were all in male subjects. Some subjects were excluded from further analysis due to a failure to participate in the 2010-2011 resurvey due to limitations of activity $(n=441)$, death during the 20102011 resurvey $(n=123)$, age greater than 90 years $(n=22)$, refusal to undergo baPWV measurements $(n=166)$, incomplete baPWV data $(n=14)$, or a history of stroke, transient ischaemic attack, and/or coronary disease $(n=$ 33). The present study therefore included 1,007 cases with CWP. This investigation also included 1,007 healthy controls from the Kailuan cohort study, all of which lacked a history of stroke, transient ischaemic attack, and coronary disease. These controls were matched to the cases based on age ( \pm 1 year), gender, systolic blood pressure $( \pm 5 \mathrm{mmHg})$, and previous history of hypertension.

\section{Diagnosis of pneumoconiosis}

All of the enrolled CWP cases had physical examination cards and detailed records of their occupational history including $\mathrm{CDE}$, individual medical and CWP diagnosis records, and measurements of dust concentrations in the subjects' workplaces, which were obtained from personnel files in the human resources section of the Kailuan Colliery Group. The diagnosis of CWP was based on the Diagnostic Criteria of Pneumoconiosis and corresponding standard videos of pneumoconiosis in China [21]. CWP was classified as stage I, stage II, or stage III according to the size, profusion, and distribution range of opacities, as previously reported [22].

\section{Dust exposure data}

Estimates of CDE were derived from each coal miner's work history up until the time of study enrolment. Work histories included job titles and calendar years for each coal worker's full duration of employment. CDE was calculated from a job-exposure matrix as follows: the duration of exposure in years was multiplied by the dust concentration at the same time in every period of dust exposure for each subject [22]. CDE is given in milligrams per cubic metre-years. Job title-specific exposure estimates were obtained from the Department of Dust Detection and Monitoring of the Kailuan Colliery Group. Dust was sampled randomly twice per month in the tunnelling, mining, combining, and helping areas. The dust concentration and free silica content were measured using the gravimetric method and the pyrophosphate method, respectively, which are national standard methods [23-25]. These numerical data were collected to calculate the geometric means of each area yearly, which were then used to calculate the CDE for each coal worker.

\section{Traditional cardiovascular risk factors}

During the resurvey in 2010-2011, all of the participants underwent a clinical examination, laboratory tests, and baPWV measurements. Structured interviews based on a standardized questionnaire were conducted by trained investigators. The questionnaire included information on the subject's demographics, history of occupational exposure, medical disorders, and traditional cardiovascular risk factors including age, smoking, body mass index, hypertension, diabetes and dyslipidaemia. Body mass index was calculated as body weight $(\mathrm{kg})$ divided by the square of body height $\left(\mathrm{m}^{2}\right)$. Current smokers were defined as subjects who had smoked at least 100 cigarettes during their lifetime and, at the time of the interview, reported smoking every day or some days. 


\section{Measurement of BaPWV}

In 2010-2011, all of the participants underwent baPWV measurements using an automatic arteriosclerosis detection device (BP-203RPE III; Omron Healthcare Co., Japan) in the supine position after at least 5 min of rest. BaPWV was calculated as the distance between the two sites divided by the pulse transit time, which was defined as the time interval between the wave front of the brachial waveform and that of the ankle waveform. The distance between the sampling points was calculated automatically according to the subject's height. The maximum value of the bilateral baPWV was used in the present analysis.

\section{Laboratory measurements}

Blood samples were obtained after at least $8 \mathrm{~h}$ of fasting and were analysed within $4 \mathrm{~h}$. Fasting blood glucose levels were measured using the hexokinase/glucose-6phosphate-dehydrogenase method. Total cholesterol and triglycerides were measured enzymatically (inter-assay coefficient of variation, 10\%; Mind Bioengineering Co. Ltd., Shanghai, China). All biochemical variables were measured using an auto-analyser (Hitachi 747; Hitachi, Tokyo, Japan) at the central laboratory of Kailuan General Hospital.

\section{Statistical analyses}

Statistical analyses were carried out using commercially available software (SAS software version 9.3; SAS Institute Inc., Cary, NC, USA). The continuous variables were described as the mean \pm standard deviation and compared using a two-sample Student's $t$-test or oneway analysis of variance for the normally distributed data. For skewed distributions, the data are presented as the median (with interquartile ranges) and compared using a Student's $t$-test or one-way analysis of variance after $\log$ transformation. The categorical variables were described as percentages and compared using the chisquared test. All tests were two-tailed and $P<0.05$ was considered to be statistically significant.

Multivariate logistic regression analyses were used to explore the association of increased baPWV with CWP and its potential risk factors after adjusting for potential confounding factors. Increased baPWV was defined as a value greater than the median baPWV of the study population. Based on similar multivariate adjustments, the odds ratios (ORs) for increased baPWV were calculated for four subject subgroups: without hypertension or CWP, with CWP only, with hypertension only, and with hypertension and CWP. Similar analyses of the relationship between increased baPWV and CDE were conducted among the CWP cases categorized into four subgroups according to the $\mathrm{CDE}$ quartile using the trend test.

\section{Results}

Basic characteristic comparisons

The demographic and clinical characteristics of the CWP cases and healthy controls are presented in Table 1 . All of the participants were male, and the mean age of the CWP cases was 65.2 years, which was similar to that of the controls $(P=0.853)$. There were no significant between-group differences in body mass index $(P=$ $0.134)$, systolic and diastolic blood pressure $(P=0.508$ and $P=0.108$, respectively), low-density lipoprotein cholesterol $(P=0.640)$, and hypertension $(P=0.646)$. The CWP cases had higher heart rates $(P<0.001)$, fasting blood glucose levels $(P=0.003)$, and triglyceride levels $(P=0.001)$, but lower total cholesterol $(P<0.001)$ and high-density lipoprotein cholesterol $(P=0.033)$ levels than the controls. Significantly fewer CWP cases were smokers $(P<0.001)$ and they were more likely to have diabetes mellitus $(P=0.051)$ than the controls. The CWP cases were categorized as follows: stage I, 980 cases (97.3\%); stage II, 22 cases (2.2\%); and stage III, 5 cases $(0.5 \%)$. The CWP cases had higher baPWV values than the controls $(P=0.006)$ and a higher proportion of the CWP cases $(P=0.016)$ had increased baPWV $(\geq 1687 \mathrm{~cm} / \mathrm{s}$ according to the median baPWV of the population) than the controls (Table 1).

\section{The association of BaPWV with CWP and traditional cardiovascular risk factors}

In the logistic regression models shown in Table 2, the CWP cases had a higher risk of increased baPWV than the controls OR 1.24 (95\% CI 1.05-1.48). After the multivariate adjustment for age, current smoking status, body mass index, heart rate, hypertension, diabetes mellitus, total cholesterol, and low-density lipoprotein cholesterol, the association remained significant OR 1.43 (95\% CI 1.11-1.83).

A further stratification analysis of the potential risk factors for baPWV (Table 3) showed that in both the CWP and control groups, age ( $\geq 60$ years), heart rate, and hypertension were positively associated with increased baPWV (all $P<0.001)$. In addition, increased baPWV was also positively associated with diabetes mellitus $(P=0.012)$ and negatively associated with current smoking status $(P<$ 0.001 ) in the control group.

\section{The combined effects of CWP and hypertension on the BaPWV}

All of the participants were categorized into four subgroups (Table 4), and the subgroup of subjects without CWP or hypertension was employed as a reference in the subsequent calculations of the ORs of increased baPWV. We detected a gradually increasing association strength across the four subgroups (P for trend $<0.001)$ as follows: CWP only, OR 1.20 (95\% CI 0.90-1.61); hypertension 
Table 1 Demographic and clinical characteristics among CWP cases and controls

\begin{tabular}{|c|c|c|c|}
\hline & CPW & Controls & $P$ \\
\hline $\mathrm{N}$ & 1007 & 1007 & \\
\hline Age (Years) & $65.15 \pm 8.86$ & $65.07 \pm 8.82$ & 0.853 \\
\hline Current smoking, n (\%) & $339(33.7)$ & 469(46.6) & $<0.001$ \\
\hline Body Mass Index $\left(\mathrm{kg} / \mathrm{m}^{2}\right)$ & $25.41 \pm 3.46$ & $25.10 \pm 3.97$ & 0.134 \\
\hline Systolic Blood Pressure (mmHg) & $138.78 \pm 18.78$ & $138.23 \pm 18.94$ & 0.508 \\
\hline Diastolic Blood Pressure (mmHg) & $85.63 \pm 10.91$ & $84.84 \pm 11.04$ & 0.108 \\
\hline Heart rate (beats/min) & $75.62 \pm 16.39$ & $71.31 \pm 11.28$ & $<0.001$ \\
\hline Fasting Plasma Glucose (mmol/L) & $5.89 \pm 1.74$ & $5.68 \pm 1.39$ & 0.003 \\
\hline Total Cholesterol (mmol/L) & $4.91 \pm 0.98$ & $5.07 \pm 1.00$ & $<0.001$ \\
\hline Low-Density Lipoprotein-Cholesterol (mmol/L) & $2.64 \pm 0.93$ & $2.67 \pm 1.00$ & 0.640 \\
\hline High-Density Lipoprotein - Cholesterol (mmol/L) & $1.52 \pm 0.48$ & $1.60 \pm 1.06$ & 0.033 \\
\hline Triglycerides $(\mathrm{mmol} / \mathrm{L})^{a}$ & $1.35(1.02,1.81)$ & $1.20(0.86,1.70)$ & $<0.001$ \\
\hline Hypertension ${ }^{\mathrm{b}}, \mathrm{n}(\%)$ & $612(60.8)$ & $612(60.8)$ & 0.646 \\
\hline Diabetes mellitus ${ }^{c}, \mathrm{n}(\%)$ & $141(14.0)$ & $111(11.0)$ & 0.051 \\
\hline \multicolumn{4}{|l|}{ Stage } \\
\hline I & $980(97.3)$ & - & \\
\hline$\|$ & $22(2.2)$ & - & \\
\hline III & $5(0.5)$ & - & \\
\hline \multicolumn{4}{|l|}{ Duration of silica dust exposure (years) } \\
\hline$<20$ & $194(19.3)$ & & \\
\hline $20 \sim 29$ & $447(44.4)$ & & \\
\hline$\geq 30$ & $366(36.3)$ & & \\
\hline Cumulative silica dust exposure $\left(\mathrm{mg} / \mathrm{m}^{3}-\mathrm{y}\right)$ & $1059.02 \pm 458$ & - & \\
\hline $\mathrm{baPWV} \mathrm{cm} / \mathrm{s}$ & $1762.0 \pm 355$ & $1718.6 \pm 354$ & 0.006 \\
\hline baPWV $\geq 1687$ cm/s, n (\%) & $533(52.9)$ & $478(47.5)$ & 0.016 \\
\hline
\end{tabular}

CWP indicates coal workers' pneumoconiosis; baPWV, brachial-ankle pulse wave velocity

${ }^{\text {a }}$ Student's $t$-test was used after log transformation

${ }^{\mathrm{b}} \mathrm{Be}$ defined as having the history of hypertension or $\mathrm{SBP} \geq 140 \mathrm{mmHg}$ or $\mathrm{DBP} \geq 90 \mathrm{mmHg}$ measured at the examination

${ }^{c} B e$ defined as having the history of diabetes mellitus or fasting blood glucose $\geq 7.0 \mathrm{mmol} / \mathrm{L}$ measured at the examination

Table 2 Logistic regression analyses of the associations of increased baPWV ( $\geq 1687 \mathrm{~cm} / \mathrm{s})$ with CWP and traditional cardiovascular risk factors among CWP cases and controls

\begin{tabular}{|c|c|c|c|c|}
\hline & \multicolumn{2}{|l|}{ Univariate model } & \multicolumn{2}{|c|}{ Multivariate model } \\
\hline & OR $(95 \% \mathrm{Cl})$ & $P$ & OR (95\%Cl) & $P$ \\
\hline Controls & 1 & & 1 & \\
\hline CWP & $1.24(1.05-1.48)$ & 0.014 & $1.43(1.11-1.83)$ & 0.005 \\
\hline Age $>=60$ years (yes vs no) & $3.48(2.87-4.22)$ & $<0.001$ & $3.39(2.62-4.30)$ & $<0.001$ \\
\hline Current smoking (yes vs no) & $0.78(0.66-0.94)$ & 0.007 & $0.96(0.76-1.22)$ & 0.760 \\
\hline Body Mass Index $\left(\mathrm{kg} / \mathrm{m}^{2}\right)$ & $0.97(0.95-1.00)$ & 0.065 & $0.95(0.92-0.98)$ & 0.002 \\
\hline Heart rate (beats/min) & $1.03(1.02-1.03)$ & $<0.001$ & $1.03(1.02-1.04)$ & $<0.001$ \\
\hline Hypertension (yes vs no) ${ }^{a}$ & $2.64(2.20-3.18)$ & $<0.001$ & $2.32(1.82-2.95)$ & $<0.001$ \\
\hline Diabetes mellitus (yes vs no) & $1.94(1.47-2.55)$ & $<0.001$ & $1.63(1.15-2.31)$ & 0.006 \\
\hline Total Cholesterol (mmol/L) & 1.08(0.99-1.18) & 0.102 & $1.11(0.98-1.27)$ & 0.106 \\
\hline Low-Density Lipoprotein-Cholesterol (mmol/L) & $0.99(0.91-1.09)$ & 0.895 & $0.94(0.82-1.07)$ & 0.335 \\
\hline
\end{tabular}

CWP indicates coal workers' pneumoconiosis; baPWV, brachial-ankle pulse wave velocity

aBe defined as having the history of hypertension or $\mathrm{SBP} \geq 140 \mathrm{mmHg}$ or $\mathrm{DBP} \geq 90 \mathrm{mmHg}$ measured at the examination

${ }^{b} \mathrm{Be}$ defined as having the history of diabetes mellitus or fasting blood glucose $\geq 7.0 \mathrm{mmol} / \mathrm{L}$ measured at the examination 
Table 3 Multivariate logistic regression analyses of the associations of increased baPW $(\geq 1687 \mathrm{~cm} / \mathrm{s})$ and traditional cardiovascular risk factors among CWP cases and controls

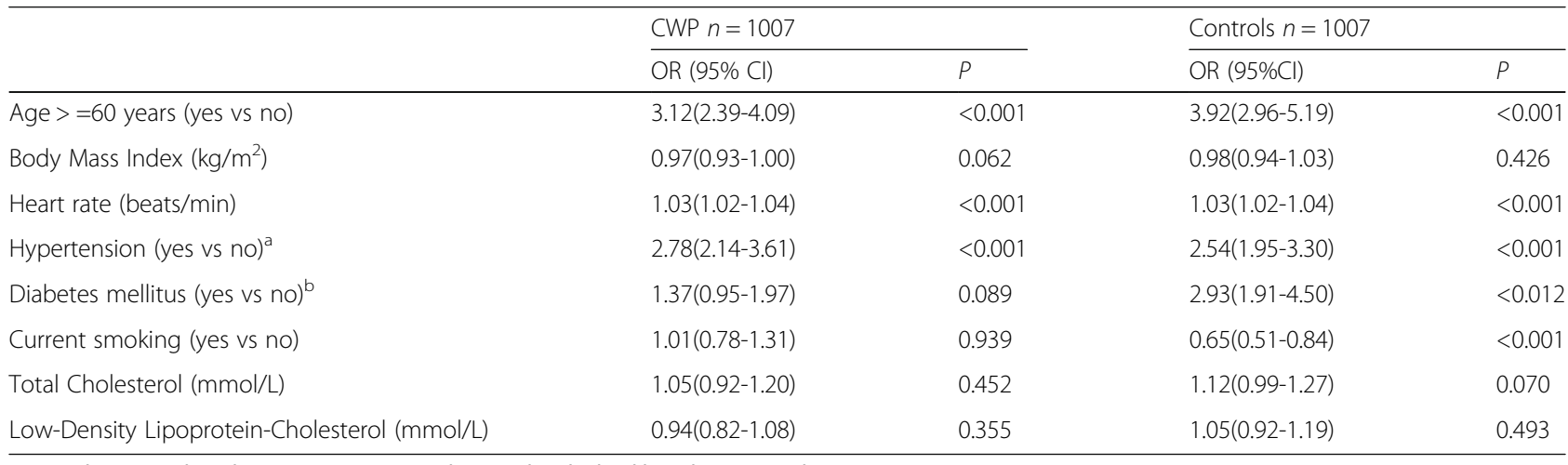

CWP indicates coal workers' pneumoconiosis; baPWV, brachial-ankle pulse wave velocity

a Be defined as having the history of hypertension or $\mathrm{SBP} \geq 140 \mathrm{mmHg}$ or $\mathrm{DBP} \geq 90 \mathrm{mmHg}$ measured at the examination

${ }^{\mathrm{b}} \mathrm{Be}$ defined as having the history of diabetes mellitus or fasting blood glucose $\geq 7.0 \mathrm{mmol} / \mathrm{L}$ measured at the examination

only, OR 2.54 (95\% CI 1.95-3.30); and both CWP and hypertension, OR 3.34 (95\% CI 2.56-4.37). The trend persisted after the multivariate adjustment ( $\mathrm{P}$ for trend, $<0.001)$, and the multivariate-adjusted ORs were 1.18 (95\% CI $0.85-1.16$ ), 1.55 (95\% CI 1.92-3.41), and 2.80 (95\% CI 2.08-3.75).

\section{The exposure-response relationship between $\mathrm{CDE}$ and BaPWV}

To explore the association between long-term CDE and increased baPWV (Fig. 1), all of the CWP cases were categorized into four subgroups according to the $\mathrm{CDE}$ quartile. We observed that the risk of increased baPWV gradually increased across the CDE quartiles, independent of traditional cardiovascular risk factors. The multivariate-adjusted ORs (compared with the cases in the first quartile) for the cases in the second, third, and fourth CDE quartiles were 1.02 (95\% CI 0.61-1.70), 1.40 (95\% CI $0.87-2.24$ ), and 2.23 (95\% CI $1.30-3.83$ ), respectively. This trend was statistically significant ( $\mathrm{P}$ for trend, 0.02). The adjusted variables included age, current smoking status, body mass index, heart rate, hypertension, diabetes mellitus, total cholesterol, and low-density lipoprotein cholesterol.

\section{Discussion}

Mortality due to CVD among coal workers with CWP is increasing $[7,8]$, which highlights the pressing need to tackle this health challenge. Early detection of CVD is key. Early detection of arterial stiffness via measurement of baPWV is useful in primary and secondary prevention of CVDs such as hypertension and coronary artery disease. Several studies have shown that baPWV is an independent predictor of the future development of CVD [7]. To the best of our knowledge, this investigation is the first demonstration of a relationship between baPWV and CWP in a relatively large sample of subjects without a history of symptomatic stroke, transient ischaemic attack, or coronary disease.

Here, the CWP cases had higher baPWV values than the controls, as well as a higher risk of increased baPWV. This risk was independent of traditional cardiovascular risk factors, including age, current smoking status, body mass index, hypertension, diabetes mellitus, total cholesterol, and low-density lipoprotein cholesterol. In this population, increased baPWV may be an independent and novel complication of CDE.

Among the CWP cases, the risk factors for baPWV included several traditional cardiovascular risk factors,

Table 4 Unadjusted and multivariate adjusted odds ratio (95\% Cl) for increased baPWV ( $\geq 1687 \mathrm{~cm} / \mathrm{s})$ among participants with CWP and/or hypertension and without $(n=2014)$ CWP indicates coal workers' pneumoconiosis; baPWV, brachial-ankle pulse wave velocity

\begin{tabular}{lllll}
\hline & Unadjusted OR $(95 \% \mathrm{Cl})$ & $P$ & Multivariate adjusted OR $(95 \% \mathrm{Cl})^{\mathrm{a}}$ & $P$ \\
\hline Without CWP and hypertension & 1 & & 1 & $1.18(0.85-1.16)$ \\
With only CWP & $1.20(0.90-1.61)$ & 0.219 & $1.55(1.92-3.41)$ & $<.313$ \\
With Only hypertension & $2.54(1.95-3.30)$ & $<0.001$ & $2.80(2.08-3.75)$ & $<0.001$ \\
With CWP and hypertension & $3.34(2.56-4.37)$ & $<0.001$ & $<0.001$ & $<0.001$ \\
$p_{\text {trend }}$ & $<0.001$ & & \\
\hline
\end{tabular}

${ }^{a}$ Adjustment for age ( $\geq 60$ years), current smoking, body mass index, diabetes mellitus, heart rate, total cholesterol, low-density lipoprotein-cholesterol 


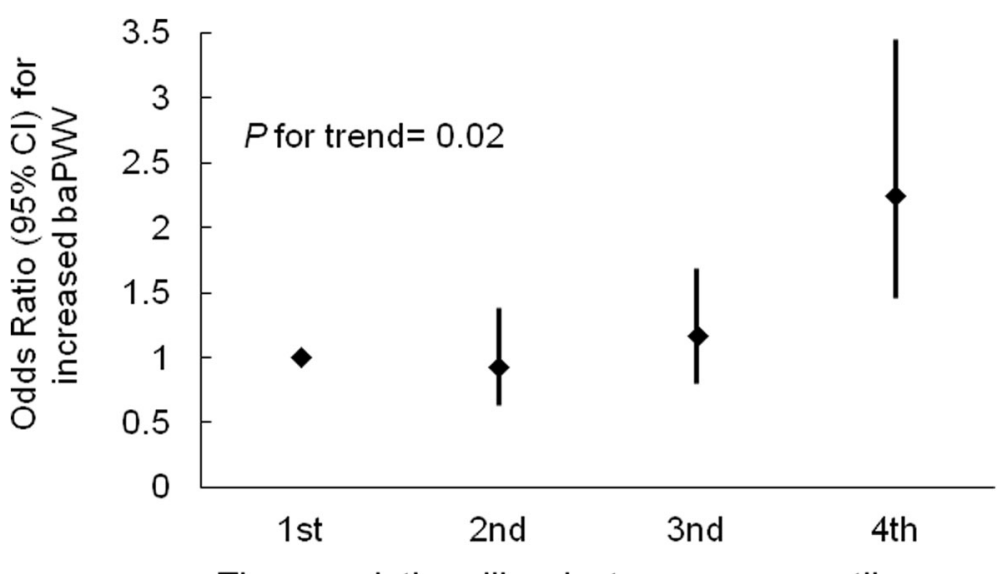

The cumulative silica dust exposure quartiles

Fig. 1 Mulitvariate Logistic regression analyses of the associations of increased baPW with cumulative dust exposure (CDE) quartiles among CWP cases. The level of CDE quartiles in all CWP cases was as follow: first, $\leq 811 \mathrm{mg} / \mathrm{m}^{3}-\mathrm{y}$; second, $811-1059 \mathrm{mg} / \mathrm{m}^{3}-\mathrm{y}$, third, $1059-1320 \mathrm{mg} / \mathrm{m}^{3}-\mathrm{y}$, and fourth, $\geq 1320 \mathrm{mg} / \mathrm{m}^{3}-\mathrm{y}$

such as age ( $\geq 60$ years), heart rate, and hypertension. In the healthy controls, baPWV was also significantly associated with diabetes mellitus. These findings agree with those of previous investigations of the general population and of patients who are at a higher risk of cardiovascular events. These investigations reported that baPWV increases with age, hypertension [26], increased heart rate [27], and diabetes [28].

However, in our multivariate analyses, no statistically significant associations of increased baPWV were found with total cholesterol and low-density lipoproteincholesterol, which might reflect selection bias in our case-control study. We enrolled a portion of the subjects from another large-scale cohort study and excluded the individuals with a history of stroke, transient ischaemic attack, and coronary diseases, which might conceal the associations that would be demonstrated in a longitudinal study. Similar reasons may explain the results of association of increased baPWV with current smoking. First, physicians would advise individuals with CWP to quit smoking for a better prognosis, which might lead to the lower prevalence of CWP cases who current smoke. As shown in Table 2, the proportion of current smoking in the CWP cases was lower than the controls $(33.7 \%$ vs. $46.6 \%, P<0.001)$. Second, our use of self-reported smoking status collected at the time of study enrolment, rather than a sensitive and specific marker of exposure to tobacco, such as cotinine, resulted in the introduction of misclassification bias into our study of smoking and baPWV. The potential for these biases should be considered when interpreting the negative association between smoking and baPWV among the controls and as well as the lack of a statistically significant association among the CWP workers.
Hypertension is a leading risk factor for CVD. Its prevalence is high and has increased rapidly over the past four decades in China, from 5\% in 1959 to $18 \%$ in $2002[29,30]$. In the present study, hypertension occurred more frequently in the CWP cases (60\%), and of the identified risk factors, hypertension had a relatively stronger effect on baPWV. Based on these considerations, we further evaluated the combined effects of CWP and hypertension on baPWV, and showed that the combined effect of these parameters increased baPWV more than either factor alone. Future studies of patients with CWP should be conducted to determine the extent to which the prevention and control of disease progression, combined with appropriate anti-hypertensive medications, can stabilize or even reverse arterial stiffness.

More importantly, the present study revealed an exposure-response relationship between $\mathrm{CDE}$ and baPWV in the CWP cases, which was independent of traditional cardiovascular risk factors. No previous reports have investigated this association. Large-scale cohort studies previously uncovered an exposure-response relationship between CDE and increased mortality from CVDs $[7,8]$. On the basis of these findings, one might hypothesize that by increasing arterial stiffness, long-term CDE increases the risk of cardiovascular events. This hypothesis should be tested in prospective cohort studies.

The mechanisms by which long-term CDE might increase arterial stiffness, as measured by baPWV, are largely unknown

Inflammation is one plausible mechanism. Atherosclerosis, a pathological process of CVD, is now generally accepted to be an inflammatory disorder of the arterial wall [31]. Inflammation may also contribute to baPWV elevation. For 
example, Saijo et al. reported a significant, progressive increase in baPWV with high-sensitivity $\mathrm{C}$-reactive protein levels in male subjects after controlling for traditional cardiovascular risk factors such as age, body mass index, systolic blood pressure, heart rate, smoking, past history of hypertension, hyperlipidaemia, and diabetes [32]. Andoh et al. also determined that baPWV is significantly associated with the serum levels of high-sensitivity Creactive protein [33]. Moreover, CWP is caused by the long-term inhalation and deposition of coalmine dust, which triggers a persistent inflammatory response and the induction of pro-inflammatory and pro-fibrotic mediators, which eventually results in irreversible lung damage [34, 35]. Respirable silica particles can initiate inflammation of the cardiovascular system via the direct effects of fine particulates that cross the pulmonary epithelium into the cardiovascular system [36] or via indirect effects mediated by the inflammatory response.

Evidence of the association between increased systemic inflammation and CWP is limited. Zhai et al. reported that the serum levels of cytokines such as interleukin 6 were associated with CWP in a Chinese sample [37]. Lee et al. suggested that high serum levels of interleukin 8 in Korean subjects were associated with CWP and those of serum tumour necrosis factor $\alpha$ were associated with the progression of CWP at the 1-year follow-up [38], but not at the 3-year follow-up [39]. In addition, studies of Chinese CWP cases revealed associations between CWP and genetic polymorphisms related to inflammatory markers such as E-selectin [40] or the inflammasome (nod-like receptor protein 3) [41]. Combining these previous data with the findings of the current investigation, it is logical to hypothesize that long-term $\mathrm{CDE}$ can instigate the inflammation response and damage arterial walls, which leads to atherosclerosis and cardiovascular events. This hypothesis needs to be validated using large-scale prospective cohort studies.

Several limitations of this study should be considered when interpreting the data. First, in light of the small sample size of the participants with CWP in Stage II and III ( $2.2 \%$ and $0.3 \%$, respectively), we could not explore the association of the severity of CWP with baPWV. Second, we were unable to elucidate the role of inflammation in the observed relationship between CWP and baPWV. Third, increased arterial stiffness is thought to increase the risk of CVD; however, baPWV only reflects the stiffness of middle-sized to large arteries and is closely correlated with carotid-femoral pulse wave velocity, which is a gold standard for the assessment of large-artery stiffness. Most importantly, the case-control design of our study hindered us from evaluating the prognostic significance of increased baPWV in CWP cases. In addition, coal dust is a mixture and other components such as polycyclic aromatic hydrocarbons might have cardiovascular effects. It is also possible that other co-exposures in the mines might contribute to the development of cardiovascular diseases. Therefore, longitudinal studies of factors that affect cardiovascular prognosis as well as studies investigating the exposure to the components of coal dust and other co-exposures in the mines are necessary in order to establish a better understanding of the risk factors of arterial stiffness and cardiovascular risk in patients with CWP.

\section{Conclusion}

In conclusion, in the present study of more than 2,000 participants, CWP was significantly associated with baPWV, independent of additional risk factors for baPWV. The combined effect of CWP and hypertension on baPWV was stronger than either factor alone. Moreover, we uncovered an exposure-response relationship between $\mathrm{CDE}$ and the risk of increased baPWV. The prognostic value of baPWV for the incidence of cardiovascular events among patients with CWP should be analysed in future prospective studies.

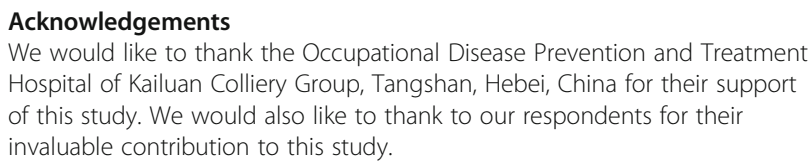

\section{Funding}

This study was funded by the Research by Kailuan (Registration No. ChiCTR-TNC-1100 1489).

\section{Availability of the data and materials}

This study is part of a largeKailuan study. The research has not concluded, and therefore, data sharing is not possible.

\section{Authors' contributions}

SW and JC conceived and designed the experiments. YZ, GY, SA, YW, and ZL performed the experiments. $Y Z$ and $L L$ analysed the data. $Z S, X Z$, and TY contributed the reagents/materials/analysis tools. $L L$ and $Y Z$ wrote the paper. All authors read and approved the final manuscript.

\section{Competing interests}

The authors declare that they have no competing interests.

\section{Consent for publication}

Not applicable.

Ethics approval and consent to participate

This study was approved by the Ethics Committees of Kailuan General Hospital and Beijing Chaoyang Hospital, China. Written informed consent was obtained from all of the participants.

\section{Author details}

${ }^{1}$ Kailuan Hospital, Tangshan, Hebei, China. ${ }^{2}$ Beijing Institute of Respiratory Medicine, Beijing Chaoyang Hospital, Capital Medical University, Beijing, China. ${ }^{3}$ Occupational Disease Prevention and Treatment Hospital of Kailuan Colliery Group, Tangshan, Hebei, China. ${ }^{4}$ Department of Cardiology, Chaoyang Hospital, Capital Medical University, Beijing, China. 


\section{References}

1. Laney AS, Wolfe AL, Petsonk EL. Pneumoconiosis and advanced occupational lung disease among surface coal miners-16 states, 2010-2011. MMWR Morb Mortal Wkly Rep. 2012;61:431-4.

2. Ross MH, Murray J. Occupational respiratory disease in mining. Occup Med (Lond). 2004:54:304-10

3. Attfield MD, Seixas NS. Prevalence of pneumoconiosis and its relationship to dust exposure in a cohort of U.S. bituminous coal miners and ex-miners. Am J Ind Med. 1995;27:37-51

4. Mo J, Wang L, Au W, Su M. Prevalence of coal workers' pneumoconiosis in China: a systematic analysis of 2001-2011 studies. Int J Hyg Environ Health. 2014;217:46-51.

5. Laney AS, Attfield MD. Coal workers' pneumoconiosis and progressive massive fibrosis are increasingly more prevalent among workers in small underground coal mines in the United States. Occup Environ Med. 2010;67:428-31.

6. Scarisbrick DA, Qunlian RM. Health surveillance for coal wokers'pneumoconiosis in the United Kingdom 1998-2000. Ann Occup Hyg. 2002;46:3.

7. Chen W, Liu Y, Wang H, Eva H, Sun Y, Su L. Long-term exposure to silica dust and risk of total and cause-specific mortality in Chinese workers: a cohort study. PLoS Med. 2012;9:e1001206.

8. Landen DD, Wassell JT, McWilliams L, Patel A. Coal dust exposure and mortality from ischemic heart disease among a cohort of U.S. coal miners. Am J Ind Med. 2011:54:727-33.

9. Zhang J, Li Y, Wang Y, Niu W, Zhang Y, Gao P, et al. Arterial stiffness and asymptomatic intracranial large arterial stenosis and calcification in hypertensive chinese. Am J Hypertens. 2011;24:304-9.

10. Tanaka H, Safar ME. Influence of lifestyle modification on arterial stiffness and wave reflections. Am J Hypertens. 2005;18:137-44.

11. Xiong Z, Zhu C, Zheng Z, Wang M, Wu Z, Chen L, et al. Relationship between arterial stiffness assessed by brachial-ankle pulse wave velocity and coronary artery disease severity assessed by the SYNTAX score. J Atheroscler Thromb. 2012:19:970-6.

12. Ninomiya T, Kojima I, Doi Y, Fukuhara Y, Hata J, Kitazono T, et al. Brachial-ankle pulse wave velocity predicts the development of cardiovascular disease in a general Japanese population: the Hisayama Study. J Hypertens. 2013;31:477-83.

13. Park KH, Park WJ, Kim MK, Jung JH, Choi HS, Cho JR, et al. Noninvasive brachial-ankle pulse wave velocity in hypertensive patients with left ventricular hypertrophy. Am J Hypertens. 2010;23:269-74.

14. Vlachopoulos C, Aznaouridis K, Terentes-Printzios D, Toakeimis N, Stefanadis C. Prediction of cardiovascular events and all-cause mortality with brachialankle elasticity index: a systematic review and meta-analysis. Hypertension. 2012;60:556-62

15. Takashima N, Turin TC, Matsui K, Rumana N, Rukamura Y, Kadota A, et al. The relationship of brachial-ankle pulse wave velocity to future cardiovascular disease events in the general Japanese population: the Takashima Study. J Hum Hypertens. 2014;28:323-7.

16. Miyano I, Nishinaga M, Takata J, Shmizu Y, Okumiya K, Matsubayashi K, et al. Association between brachial-ankle pulse wave velocity and 3-year mortality in community-dwelling older adults. Hypertens Res. 2010;33:678-82.

17. Matsuoka O, Otsuka K, Murakami S, Hotta N, Yamanka G, Kubo Y, et al. Arterial stiffness independently predicts cardiovascular events in an elderly community - Longitudinal Investigation for the Longevity and Aging in Hokkaido County (LILAC) study. Biomed Pharmacother. 2005;59 Suppl 1:S40-4.

18. Wu S, Huang Z, Yang X, Zhou Y, Wang A, Chen L, et al. Prevalence of ideal cardiovascular health and its relationship with the 4-year cardiovascular events in a northern Chinese industrial city. Circ Cardiovasc Qual Outcomes. 2012:5:487-93.

19. Wang F, Wu S, Song Y, Tang X, Marshall R, Liang M, et al. Waist circumference, body mass index and waist to hip ratio for prediction of the metabolic syndrome in Chinese. Nutr Metab Cardiovasc Dis. 2009;19:542-7.

20. Wu S, Li Y, Jin C, Yang P, Li D, Li H, et al. Intra-individual variability of highsensitivity C-reactive protein in Chinese general population. Int J Cardiol. 2012;157:75-9.

21. National Health and Family Planning Commission of the People's Republic of China (2009) Diagnostic criteria of pneumoconiosis. Occupational health standard of the people's Republic of China, GBZ 70-2009

22. Shen F, Yuan J, Sun Z, et al. Risk identification and prediction of coal workers' pneumoconiosis in Kailuan Colliery Group in China: a historical cohort study. PLOS ONE. 2013;8:e82181.

23. National Health and Family Planning Commission of the People's Republic of China (2007) Method for determination of dust in the air of workplace, Part 1, Total dust concentration,GBZT 192.1-2007.
24. National Health and Family Planning Commission of the People's Republic of China (2007) Method for determination of dust in the air of workplace, Part 2, Respiratory dust concentration,GBZT 192.2-2007

25. National Health and Family Planning Commission of the People's Republic of China (2007) Method for determination of dust in the air of workplace, Part 4: Content of free silica in dust.,GBZT 192.4-2007.

26. Fujiwara Y, Chaves P, Takahashi R, Amano H, Kumagai S, Fujita K. Relationships between brachial-ankle pulse wave velocity and conventional atherosclerotic risk factors in community-dwelling people. Prev Med. 2004;39:1135-42.

27. Tomiyama H, Hashimoto H, Tanaka H, Matsumoto C, Odaira M, Yamada J, et al. Synergistic relationship between changes in the pulse wave velocity and changes in the heart rate in middle-aged Japanese adults: a prospective study. J Hypertens. 2010;28:687-94.

28. Ohnishi H, Saitoh S, Takagi S, Ohata J, Isobe T, Kikuchi Y, et al. Pulse wave velocity as an indicator of atherosclerosis in impaired fasting glucose: the Tanno and Sobetsu study. Diabetes Care. 2003;26:437-40.

29. Wang LD. Nutrition and health status in Chinese people. Beijing (CN): People's Publishing House; 2005

30. Huang JS. he summary report of the National Symposium on Heart and Vascular Diseases. The collection of scientific reports on the National Symposium on Heart and Vascular Diseases. Beijing (CN): People's Publishing House; 1960.

31. Ross R. Atherosclerosis-an inflammatory disease. N Engl J Med. 1999;340:115-26.

32. Saijo Y, Utsugi M, Yoshioka E, Horikawa N, Sato T, Gong Y. Relationships of C-reactive protein, uric acid, and glomerular filtration rate to arterial stiffness in Japanese subjects. J Hum Hypertens. 2005:19:907-13.

33. Andoh N, Minami J, Ishimitsu T, Ohrui M, Matsuoka H. Relationship between markers of inflammation and brachial-ankle pulse wave velocity in Japanese men. Int Heart J. 2006;47:409-20.

34. Nawrot TS, Alfaro-Moreno E, Nemery B. Update in occupational and environmental respiratory disease 2007. Am J Respir Crit Care Med. 2008; 177:696-700.

35. Huang X, Li W, Attfield MD, Nadas A, Frenkel K, Finkelman R. Mapping and prediction of coal workers' pneumoconiosis with bioavailable iron content in the bituminous coals. Environ Health Perspect. 2005:113:964-8.

36. Nemmar A, Hoet PH, Vanquickenborne B, Dindale D, Thomeer M, Hoylaerts MF, et al. Passage of inhaled particles into the blood circulation in humans. Circulation. 2002;105:411-4

37. Zhai R, Liu G, Ge X, Bao W, Wu C, Yang C, et al. Serum levels of tumor necrosis factor-alpha (TNF-alpha), interleukin 6 (IL-6), and their soluble receptors in coal workers' pneumoconiosis. Respir Med. 2002;96:829-34

38. Lee JS, Shin JH, Lee JO, Kim JH, Lee KM, Choi BS, et al. Serum Levels of Interleukin-8 and Tumor Necrosis Factor-alpha in Coal Workers' Pneumoconiosis: One-year Follow-up Study. Saf Health Work. 2010:1:69-79.

39. Lee JS, Shin JH, Lee KM, Hwang JH, Baek JE, Kim JH, Choi BS. Serum levels of TGF-beta1 and MCP-1 as biomarkers for progressive coal workers' pneumoconiosis in retired coal workers: a three-year follow-up study. Ind Health. 2014;52:129-36.

40. Wang T, Ji X, Luo C, Fan J, Hou Z, Chen M, et al. Polymorphisms in SELE gene and risk of coal workers' pneumoconiosis in Chinese: a case-control study. PLOS ONE. 2013;8:e73254.

41. Ji X, Hou Z, Wang T, Jin K, Fan J, Luo C, et al. Polymorphisms in inflammasome genes and risk of coal workers' pneumoconiosis in a Chinese population. PLoS ONE. 2012;7:e47949.

\section{Submit your next manuscript to BioMed Central and we will help you at every step:}

- We accept pre-submission inquiries

- Our selector tool helps you to find the most relevant journal

- We provide round the clock customer support

- Convenient online submission

- Thorough peer review

- Inclusion in PubMed and all major indexing services

- Maximum visibility for your research

Submit your manuscript at www.biomedcentral.com/submit 